\title{
Article
}

\section{Strain ratio as a quantification tool in strain imaging}

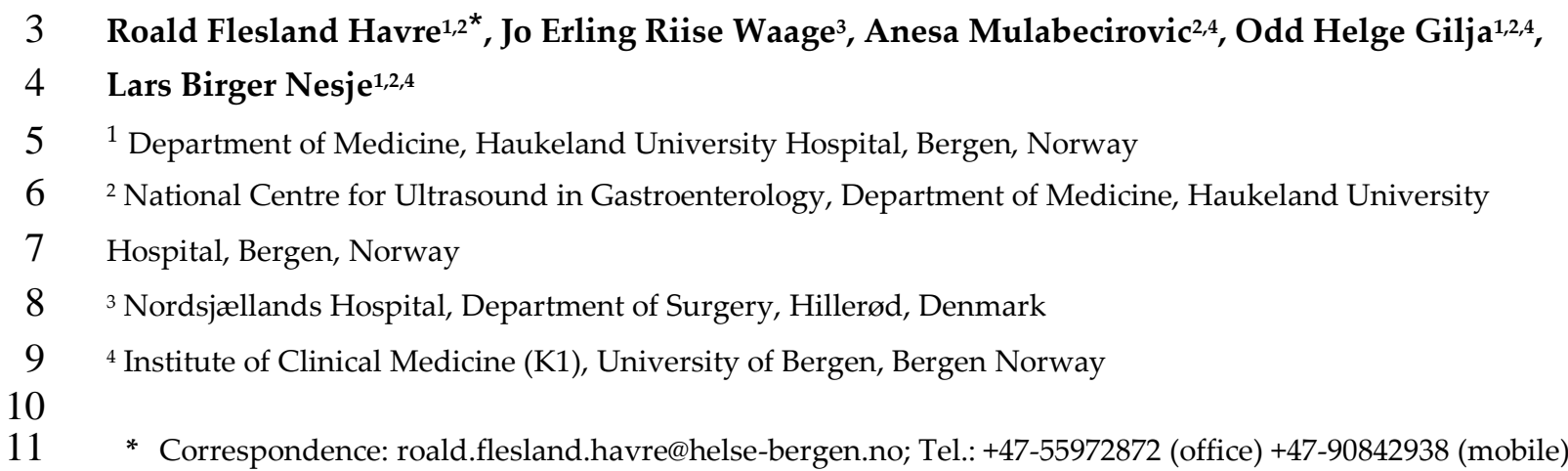

Featured Application: Strain based elastography. In this paper the system used is Real-Time Elastography, (Hitachi Medical Corporation, Zug, Switzerland) with relevant probes for external and endoscopic applications.

\section{Abstract:}

1) Background: Ultrasound-based strain imaging is now available in several ultrasound (US) scanners. Strain ratio (SR) can be used to quantify strain recorded simultaneously in two different user-selected areas, ideally exposed to the same amount of stress. The aim of this study was to evaluate SR variability when assessed in an in-vitro setup with a tissue-mimicking phantom, on resected tissue samples and in live tissue scanning with endoscopic applications. 2) Material and methods: Retrospective analysis of SR for quantification of elastic contrasts in a tissue-mimicking phantom containing four homogenous inclusions, in 38 resected bowel wall lesions and in 48 focal pancreatic lesions examined in vivo. Median SR and the inter-quartile range (IQR) was calculated on all external and endoscopic US-applications. The IQR/median provides a measure of the SR variability focusing on the two percentiles of the data closest to the median value. 3) Results: The overall variability of SR was lowest in a tissue-mimicking phantom (mean QR/median SR: 0.07). In resected bowel wall lesions representing adenomas, adenocarcinomas or Crohn lesions, the variability increased (mean IQR/Median: 0.62). During an endoscopic examination of focal pancreatic lesions in vivo, the variability increased further (mean IQR/Median: 2.04). 4) Conclusion: SR variability increased when assessed on different targets with growing heterogeneity and biological variability as one moved from homogeneous media to live tissues and endoscopic application. This may indicate a limitation for the accuracy SR evaluation in clinical applications.

34 Keywords: Ultrasound, strain elastography, quantification, strain ratio, strain quantification, 


\section{Introduction}

Soft tissue elastic properties change in various pathological tissues such as malignant tumors and inflammatory processes. Strain elastography can be used to quantify this physical feature based on ultrasound imaging [1]. Tissue hardness can be assessed across tissue images describing the Elastic modulus (E), defined as the relationship between the application of local stress and the resulting strain. This can be expressed as:

$$
E=\frac{\Delta \text { strain }}{\Delta \text { stress }}
$$

Since the stress is not recorded as it travels from the stress source through the tissue and gradually attenuates, it is not possible to calculate the Elastic modulus from strain data alone. This phenomenon is sometimes referred to as the "inverse problem" of strain elastography [2]. Under similar stress, strain in harder tissue is lower than strain in softer tissue. Thus, a comparison between strain in reference tissue and in the lesion, produces a ratio that increases when the focal lesion is harder. SR represents the relative difference in tissue hardness and was originally introduced as the "Fat lesion ratio" [3]. The definition of SR is:

$$
\text { Strain Ratio }(S R)=\frac{\text { Mean strain } B(\text { reference area })}{\text { Mean strain } A(\text { lesion } \text { area })}
$$

Hooke's law states that for small deformations in elastic media the strain is linearly proportional with the force (stress) applied to it. However, this is true for isotropic, homogeneous media with near infinite or free border conditions [4]. These conditions are rarely present in biological tissues, which exhibit non-linear elastic properties of different magnitude due to differences in tissue structure and function $[5,6,7]$. This may be of importance for the accuracy of strain elastography. By keeping the pre-compression and range of compressions ( $\Delta$ Stress) to a limited interval, the stress-strain relation of the tissues involved may for practical purposes be regarded as linear.

Vital tissue contains ducts and veins acting as dampers of stress as well as connective tissue and sliding anatomical surfaces that limit and enhance tissue movement, respectively. Unintended movements may cause strain concentration and reduce accuracy in SR evaluation. Hence, in-vivo conditions often do not meet the preconditions of Hooke's law for elasticity calculation, and may therefore represent limitations and cause strain-imaging artefacts that increases variability and probably reduce the reproducibility of SR measurements.

SR expresses a momentarily and relative difference in compressibility in two user selected areas within selected regions of interest in a strain elastogram. SR depends on similar stress application to the two areas compared and similar stress attenuation in the tissue between the stress source (probe) and the area of interest [8]. The SR measurement method was first introduced as the "Fat-lesion-ratio" in breast imaging, where an area of subcutaneous fat was used as reference to mean strain in the lesion under investigation [3]. Using the subcutaneous fat as reference was perhaps as close to a standardized reference tissue one can get. However, the same preconditions apply as the position and probe distance to a hard or soft lesion may influence the strain distribution in reference area fat tissue [8].

The variations in elasticity of biological tissues is not linear with varying pre-compression or stretch of the tissue [9]. For breast and pancreatic tissues this has been evaluated by force 
indentation and deformation studies under increasing strengths $[5,6]$. This implies that the amount of pre-compression and the range of the stress applied influences the strain result, and thereby the elastogram. In one study, the authors recommend a pre-compression level less than 0.2-0.4 $\mathrm{kPa}$ in breast imaging [6].

Another physical condition that complicates reproducibility of strain ratios is the temporal variability of a live strain cine-loop [10]. The best phases for acquiring strain data is during the compression and decompression phase, since there is no strain signal when the stress is stable between these two phases. Compression and decompression of vital tissue may be caused by applied pressure from the probe or by natural internal movements from arterial pulsation, heart movements or even breathing. Some strain elastography platforms provide feedback to the examiner about the phase of compression or decompression on which the image is acquired, enabling the use of SR from similar phases of tissue straining.

The aim of this study was to retrospectively compare SR variability as expressed by the median value and interquartile range (IQR) recorded in homogeneous tissue-mimicking media, in resected tissue from bowel lesions and during endosonography of focal pancreatic lesions.

\section{Materials and Methods}

The SR data in this study were recorded on different versions of the Hitachi Medical Corporations Real-Time Elastography (RTE) operated on HV-900 and Ascendus platforms. US data were acquired using external linear probes (L54, 9-13 MHz) as well as Pentax echo endoscope EG-3870 UTK (Pentax Medical, Hamburg, Germany). The phantom used is a standard model made of Zerdine ${ }^{\circledR}$ embedded in a firm box including eight spherical inclusions with elasticity $8 \mathrm{kPa}, 14$ $\mathrm{kPa}, 45 \mathrm{kPa}$ and $80 \mathrm{kPa}$ in a background of $25 \mathrm{kPa}$ (CIRS, model 49, NC, USA).

\section{Statistical Methods}

We used the mean of median SR values for each class of lesion, the range of values (max-min) and the interquartile range (IQR) for different objects or lesions. The IQR is a measure of the variability based on the two central quartiles from $25-75$ percentile. The remaining $50 \%$ in the eccentric quartiles, are not part of the IQR, but are encountered for by the range, representing the gap between the highest and lowest measured value. Kolmogorov-Smirnov's test was used to determine the distribution of data, and one way ANOVA or non-parametric tests were used accordingly. We then used the Kruskal- Wallis test for individual samples to compare the Median SR, the IQR and the IQR/Median for the three applications of Real-Time elastography with SR. We also analyzed the difference for Median SR, IQR and IQR/Median between observer A and B in the phantom and for benign or malignant pancreatic lesions by EUS elastography using one way ANOVA and T-test. A difference with $p$-value $<0.05$ was considered statistically significant.

All patients had signed a consent form to participate in the two studies that provided SR data for comparison of variability. For statistical analysis, we used SPSS, version 24 (SPSS, IBM, NY, USA). Study protocols as well as patient information \& consent forms were approved by the institutional committee for Research in Medicine and Biology. The studies were conducted according to the Helsinki Declaration for Research in Medicine and Biology.

\section{Results}

In a homogeneous, tissue-mimicking phantom, four spherical inclusions with elasticity different from the background were examined by two different observers. Observer A had little experience with US scanning and observer $B$ had long experience with both phantom and clinical application of US strain imaging methods. An image of inclusion $4(80 \mathrm{kPa}+/-12 \mathrm{kPa})$ is shown in Fig 1. 


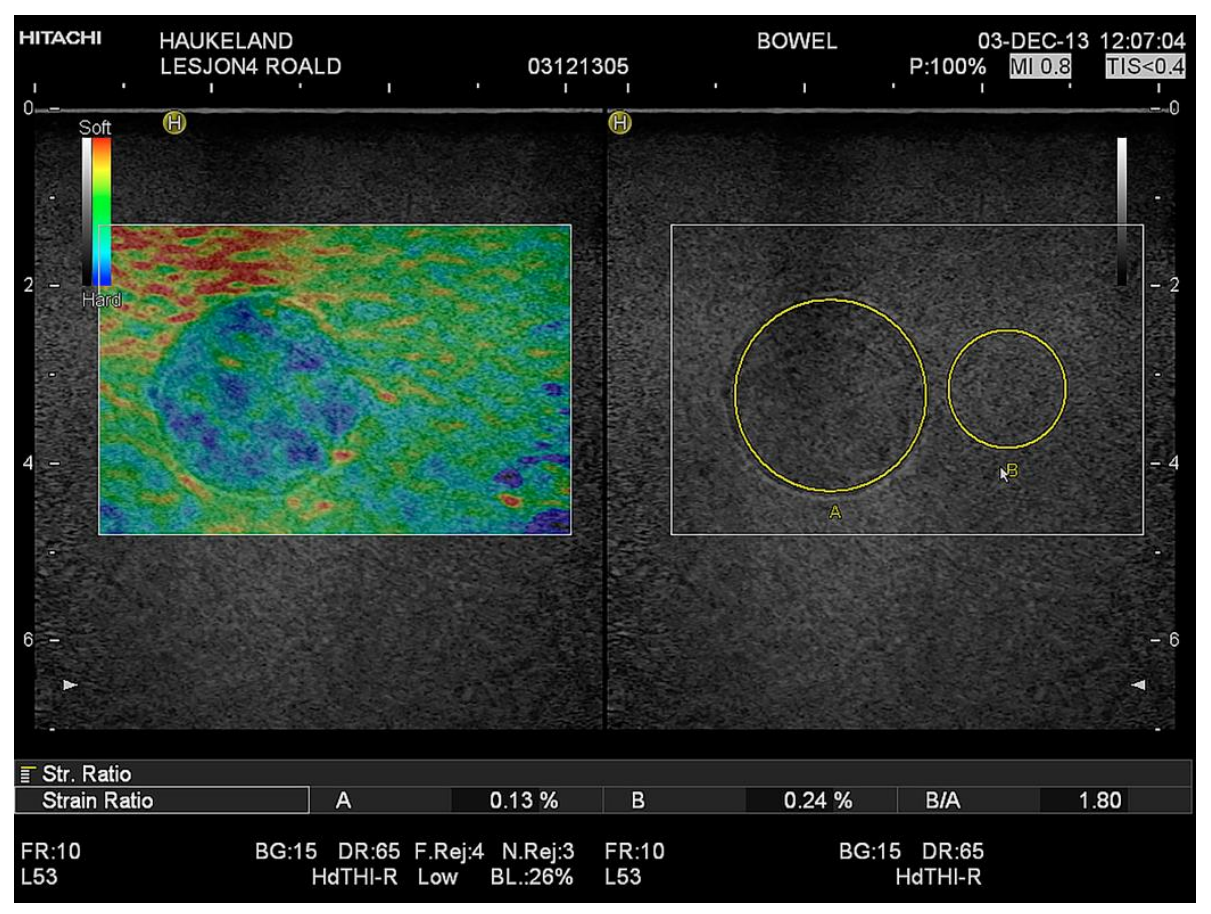

136 Fig 1: Elastogram from a tissue-mimicking phantom displaying a spherical inclusion (80kPa) with a 137 diameter of $2 \mathrm{~cm}$ in a background of $25 \mathrm{kPa}$. Right side: B-mode image with area markings; A (lesion) 138 and $B$ (reference). Left side: elastogram in color coding. The stress source was working from above in 139 the axial direction. The lesion is speckled, green-blue while the background material is mostly

140 homogeneously green. Between the lesion and the probe the red color indicates strain concentration 141 between the stress source and the harder lesion. The Strain ratio is Mean strain in B / Mean strain in $142 \quad A=1.80$.

143 In Table 1 the median values of ten repeated SR measurements are reported for observer A and B, 144 their range and interquartile range (IQR) and the IQR/median. The last measure represents the 145 variability of $50 \%$ of the central observations divided by the median value. The common mean value 146 for observer A and B was 0.07. IQR for all median SR values for all inclusions was $\leq 0.17$. 
158

159 160
161

162

163

164

165

166

167

168

169

170

171

172

173

174

175

176

177

178

179

180

181

182

183

Table 1. Elastography strain ratio in four inclusions in a tissue-mimicking phantom

\begin{tabular}{|c|c|c|c|c|c|c|c|c|}
\hline $\begin{array}{l}\text { Lesion } \\
\text { Background } 25 \mathrm{kPa}\end{array}$ & \multicolumn{2}{|l|}{1} & \multicolumn{2}{|c|}{$\begin{array}{l}2 \\
14 \mathrm{kPa}\end{array}$} & \multicolumn{2}{|c|}{$\begin{array}{l}3 \\
45 \mathrm{kPa}\end{array}$} & \multicolumn{2}{|c|}{$\begin{array}{l}4 \\
80 \mathrm{kPa}\end{array}$} \\
\hline Observer & $\mathrm{A}$ & B & $\mathrm{A}$ & B & $\mathrm{A}$ & B & $\mathrm{A}$ & B \\
\hline Median SR & 0.52 & 0.68 & 0.96 & 0.82 & 1.91 & 1.35 & 2.50 & 2.82 \\
\hline Range & 0.09 & 0.08 & 1.04 & 0.10 & 0.18 & 0.11 & 0.91 & 0.24 \\
\hline $\mathrm{IQR}^{1}$ 25-75 & 0.06 & 0.03 & 0.15 & 0.06 & 0.12 & 0.04 & 0.17 & 0.07 \\
\hline IQR/Median & 0.118 & 0.044 & 0.156 & 0.073 & 0.063 & 0.030 & 0.068 & 0.025 \\
\hline
\end{tabular}

${ }^{1}$ IQR: Inter Quartile Range. Mean IQR/median for phantom lesions: 0.07

\section{Interobserver variability}

The interobserver variability in the phantom lesions expressed by the mean of SR medians showed no significant difference for the two observers ( $\mathrm{p}=0.937$ ANOVA). The IQR/Median SR ranged from 0.063-0.156 (Mean: 0.101) for observer A with least experience, and 0.025-0.073 (Mean 0.043 ) for observer $B$ with more experience, but the difference was not significant ( $p=0.055$ ANOVA).

The distributions of Median SR and IQR/median were not significantly different between observer A and B. The mean IQR alone was significantly different between observer A: 0.125 (SD:0.050) and observer B 0.050 (SD:0.018) ( $\mathrm{p}=0.027$ ANOVA). Range was not significantly different between the observers.

\section{Strain ratio in surgical specimens}

One observer scanned surgically removed bowel specimens including tumors or resected Crohn lesions. The image of a scanned bowel wall with an adenocarcinoma is shown in Fig 2. SR was recorded between normal bowel wall and peri-colic fat/ connective tissue and the lesion of interest. SR results including range, IQR and IQR/median for the entities adenoma, Crohn lesions and adenocarcinomas are reported in Table 2. Crohn lesions had a very wide range in measurements (21.44), but this entity had the lowest IQR/Median: 0.31. For adenocarcinomas, the IQR/Median was 0.66 and for adenomas, represented by a very limited number (4), the IQR/Median was 0.88 . For all SR measurement in resected bowel tissue the variability expressed by IQR was $\leq 0.88$. The mean IQR/Mean in all resected tissue was 0.62 . 


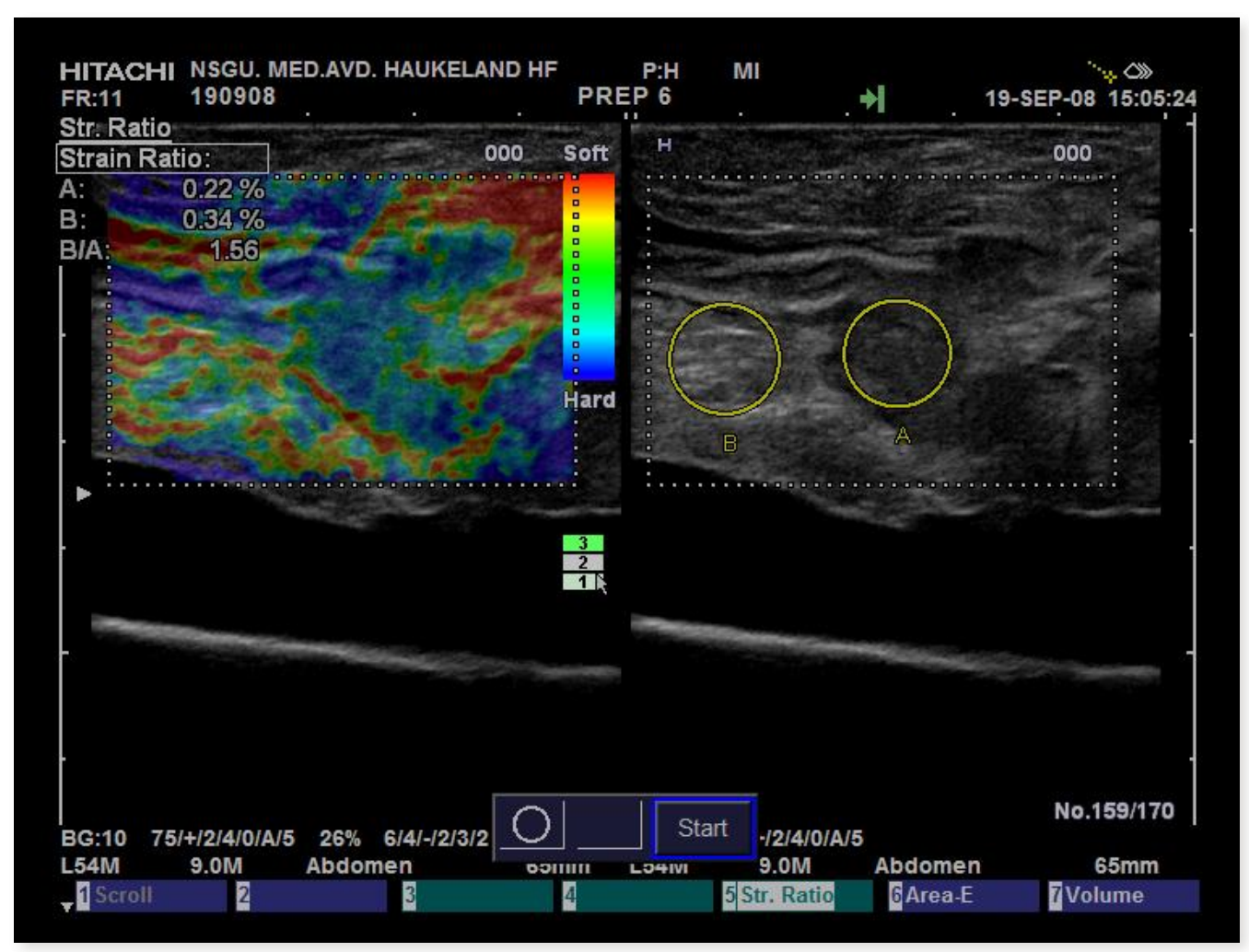

184

Fig 2: Elastogram of a newly resected bowel lesion from the colon containing a malignant tumor (adenocarcinoma). The hypoechoic tumor mass (right) is imaged with a blue-green color indicating harder tissue (left). A strain ratio measured between pericolic fat and connective tissue as well as part of the proper muscle and the tumor tissue yields $S R=1.56$. The lesion and reference are positioned at similar depth and distance from the stress source and the bottom.

Table 2. Elastography Strain ratio in resected surgical bowel specimens

\begin{tabular}{|l|l|l|l|}
\hline & Adenoma & Crohn & Adenocarcinoma \\
\hline Number & 4 & 16 & 18 \\
\hline Median SR & 1.25 & 2.09 & 2.18 \\
\hline Range & 1.38 & 21.44 & 4.53 \\
\hline IQR $^{1}$ (25-75) & 1.10 & 0.64 & 1.44 \\
\hline IQR/Median & 0.88 & 0.31 & 0.66 \\
\hline
\end{tabular}

${ }^{1}$ IQR: Inter Quartile Range. Mean IQR/Median for ex vivo tissue: 0.62

\section{Strain ratios in live tissue using endosonography}

196 The data on pancreatic lesions representing various focal entities is reported in Table 3. The mean and range of

197 these data has previously been published [11]. The mean of Median SR of malignant pancreatic lesions was 1987.05 (SD 1.85) and for the benign lesions it was 2.15 (SD 1.22), (p=0.035 T-test). For all entities, the IQR was 
199 higher than the median value of SR, indicating substantial variability. For the malignant lesions the mean 200 IQR/Median SR was 1.79 (SD 0.69). For the benign lesions the mean IQR/Median SR was 2.21 (SD 1.29). The 201 difference was not significant ( $\mathrm{p}=0.713$, T-test). The mean IQR/Median SR for all pancreatic focal lesions were 202 2.04. The IQR/Median SR value for lesions in focal pancreatitis was the highest value for all lesions, reflecting 203 the large variability observed in focal pancreatitis as well as a relatively low Median SR for this entity (0.91).

Table 3. Elastography strain ratio of focal pancreatic lesions by Endoscopic Ultrasound

\begin{tabular}{|l|l|l|l|l|l|}
\hline Entity & NET & NET & Adenocarcin & Focal & Other benign \\
\hline or benign & malignant & oma & pancreatitis & lesion \\
\hline Number & 11 & 7 & 11 & 8 & 11 \\
\hline Median SR & 2.19 & 5.74 & 8.36 & 0.91 & 3.34 \\
\hline Range & 7.93 & 17.5 & 24.5 & 8.33 & 35.3 \\
\hline IQR ${ }^{1}$ (25-75) & 2.80 & 13.1 & 10.9 & 3.55 & 5.53 \\
\hline IQR/Median & 1.28 & 2.28 & 1.30 & 3.68 & 1.66 \\
\hline
\end{tabular}

IIQR: Inter Quartile Range. Mean IQR/Median for pancreatic lesions: 2.04

IQR/Median SR for three applications of strain elastography

For the three applications reported here the IQR/median SR increased from scanning a tissue mimicking phantom to ex-vivo surgical specimens of bowel pathology and further when the strain imaging was performed endoscopically focusing on focal pancreatic lesions. The difference between the IQR/Median SR was significant ( $\mathrm{p}=0.002$, Kruskal-Wallis) Fig. 3. 


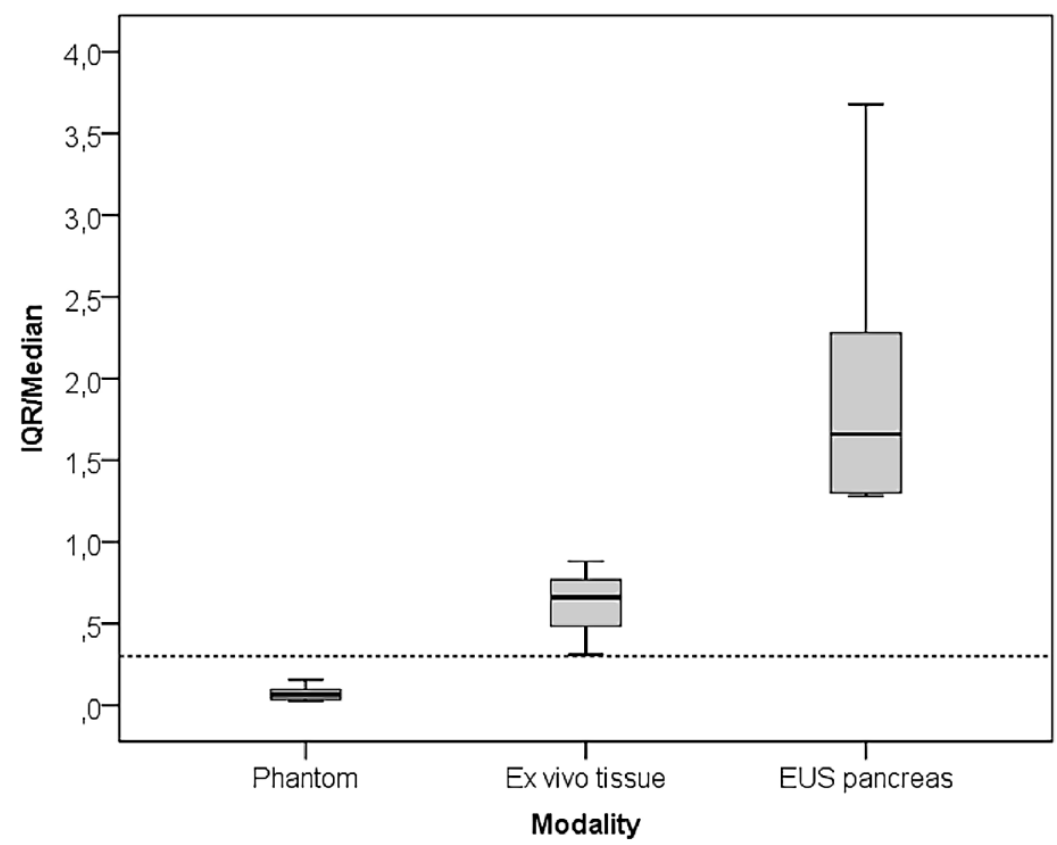

Fig. 3: Box plots of the IQR/Median SR for the different applications of strain based elastography (Real-Time Elastography) reported here. There is significant difference between this quality parameter for the three applications $(p=0.002)$. In liver elastography using Transient Elastography, the suggested maximum $I Q R /$ Median for good quality assessment in 10 repeated measurements in the same liver is 0.30 , which is marked with the dotted horizontal line.

\section{Reference area variability}

Fig. 4 demonstrates three different frames (A-C) of strain images by EUS elastography including SR measurements of the same pancreatic tumor using slightly different, but relevant reference areas. The three SRs obtained range from $8.43-16.36-25.70$. All the variation is caused by variability in the reference tissue, in which strain varies between $0.08 \%-0.16 \%-0.27 \%$. The lesion

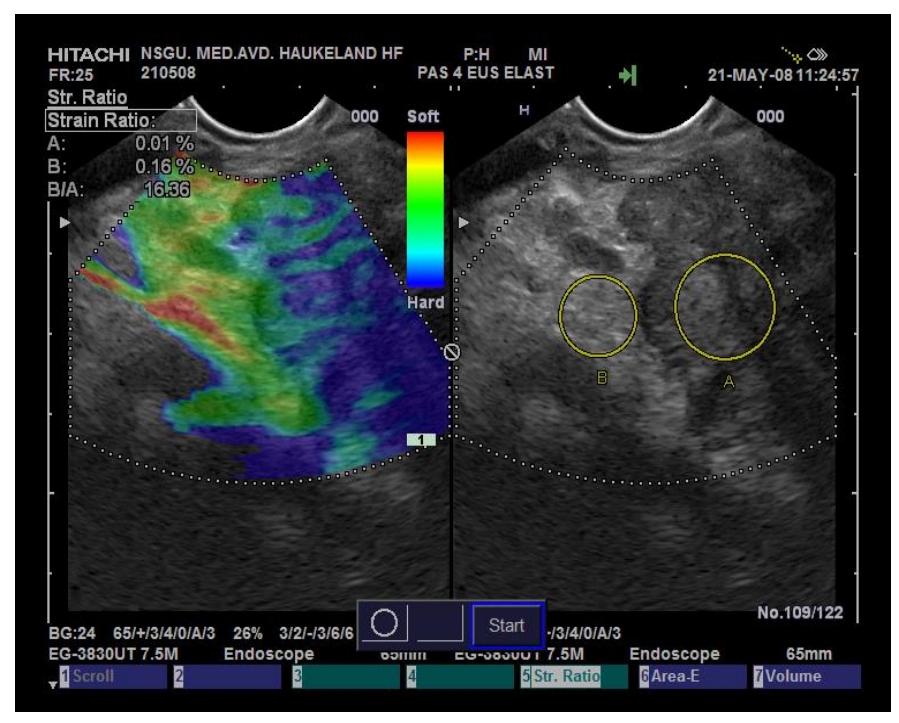



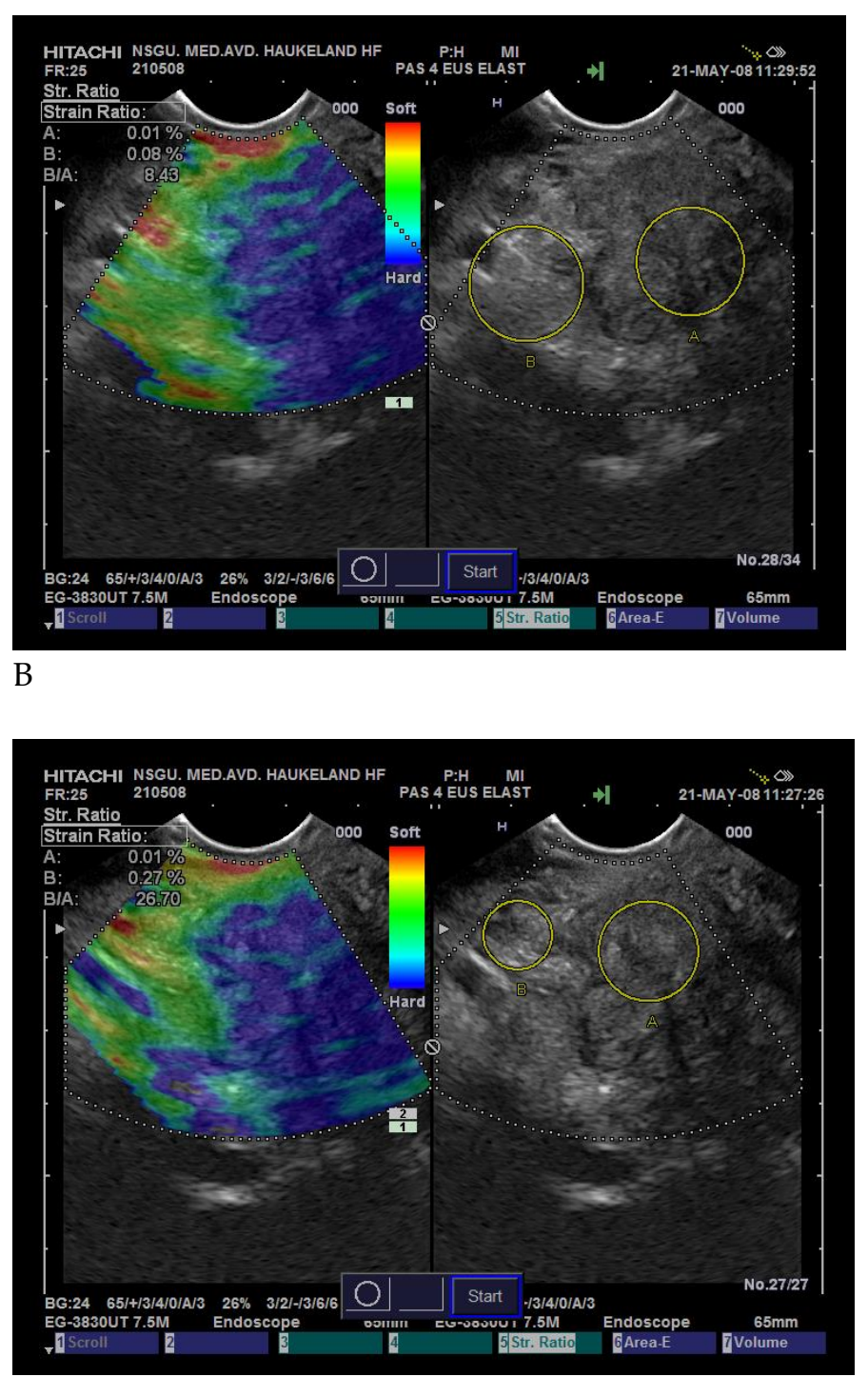

Fig. 4: Three images of the same pancreatic tumor visualized by EUS elastography. Tumor tissue is blue in the elastograms with a predominantly green reference tissue. The position and size of the reference tissue vary slightly between the three different images, and exhibit different strains in all three images, A: 0.16\% B: 0.08\% and C: $0.27 \%$, while the strain in the lesion remains $0.01 \%$. This causes the SR values to vary from $8.43-26.7$.

\section{Discussion}

In this paper, we have presented SR measurement in a tissue-mimicking phantom and in resected tissue where the stress is applied by pushing gently with the probe itself focusing on the measurement variability. We have also presented data from endosonography of focal pancreatic lesions where arterial pulsations, particularly from the aorta, acts as an internal stress source. We have shown that the IQR increases with complexity and anisotropy in the tissues to a level that surpasses the median value of SR. The mean value in of the IQR/Median increases 8,6 times from phantom (0.07) to resected tissue (0.62) and 28,3 times to endosonographic application (2.04).

Selection of the reference area for comparison with a focal lesion also introduce variability which may influence the equation and the resulting SR more than the strain variability of the lesion itself. (Fig 4). Some researchers have only recorded the strain value in the lesion of interest, and found that it performs equally well as the strain ratio in some applications $[12,13]$. This however, is dependent on a near standardized application of stress to the lesions of interest. But usually the amount of stress cannot be sufficiently standardized, and depends on type of application, lesion and 
patient related factors such a depth of lesion and elasticity of surrounding tissue. For the endoscopic application, also the pulse pressure, the aortic compliance and the presence of peri-pancreatic soft tissue would be different from patient to patient. Therefore, a standardization of the stress applied to the lesion of interest in endoscopic strain elastography is almost impossible to establish. Moreover, further differences may be caused by variable levels of pre-compression and applied stress. Since soft tissues have non-linear elastic properties, the tissue will appear harder with more applied stress or increased pre-compression [5] [6]. On the other hand, this strain-stress profile under different levels of stress may be a valuable signature of the different soft tissue, including pathological lesions, and may be used for tissue characterization if a series of graded stress pulses may be applied to the ROI.

Another way to quantify a strain image is by making a strain histogram [14]. This shows the distribution of the different colors representing different strain intervals in a 256 scale, from no strain (0) to max strain as set by the scanner software (256). From a histogram it is also possible to quantify the distribution of recorded strains to evaluate if the lesion and reference tissue is homogeneous or heterogeneous by using kurtosis and range. The median value of the histogram represents the median hardness of the lesion. Some researchers have suggested to use median histogram value of reference tissue divided by the median value of the lesion histogram to create a "histogram ratio". In a study of pancreatic lesions, this did not perform better than SR with a ROC-AUC of 0.843 and a cut-off selection yielding a specificity of $98 \%$, and a sensitivity of only $50 \%$. (accuracy of $69 \%$ ) [15].

Visual evaluation of the elastogram is direct and intuitive and requires no post-processing. Several scoring systems for categorical scoring of strain images have been proposed. For breast imaging the 5-point Tsukuba scale was proposed by Itoh et al. in 2006 [16] . Amended versions of this visual score have been proposed for other organs, e.g. the pancreas and lymph nodes by EUS elastography [17]. Both continuous VAS score and categorical visual scores for endoscopic assessment of rectal tumors has shown comparable results with SR measurements [18]. In a comparative study of SR and the 5-point visual elastography breast scale, SR came out with better accuracy [19]. The direct visual impression the experienced examiner gets by elastography imaging, however, may contain more information than a 5-step visual score can comprehend, and is useful in many cases for lesions in doubt. With training, different aspects of the elastogram such as strain concentration and artefacts caused by veins and natural sliding surfaces can be recognized. These findings can hardly be recognized by SR, histograms or other formal quantification methods. One group has investigated the use of automated pattern- and color recognition of elastography of focal pancreatic lesions in 258 patients. They used an artificial neural network image- analysis and information about the histological diagnosis from endoscopic elastography as input. This improved the accuracy by ROC-AUC to 0.94, significantly better compared to using only lesion strain histograms that had a ROC-AUC of 0.85 . This evaluation was done from material in a multicenter study [20] but required substantial post-processing.

Using an endoscopic application in live tissue, the stress is mainly caused by arterial pulsations, heartbeat or breathing. An endoscopic application is also challenging because the probe is inserted into the gastrointestinal cavity, and cannot be controlled directly by the observer's hands, in the same manner as in external strain imaging ultrasonography. Lu and Chen et al. performed a meta-analysis of EUS elastography in pancreatic lesions evaluated qualitatively by a visual score, by strain histograms, SR, contrast enhanced EUS and EUS FNA [21]. They identified a large range in SR cut-off values based on previous published cut-off values or the ROC curves (3.05 to 24.82). This was reason to large heterogeneity for the specificity, and $3 / 8$ studies were identified as outliers. After removing the outliers, the evaluation for identification of malignant lesions based on qualitative visual scores and strain histograms outperformed the SR based evaluation (sens: 0.94, spes: 0.54, Diagnostic OR: 29.42, Q: 0.812). On the other hand, strain elastography and SR based assessment of rectal tumors using a dedicated radial rectal probe has been used to improve selection of patients for organ-sparing treatment compared to standard multidisciplinary assessment [22]. 


\section{Limitations:}

This study is based on previously recorded strain ratios from a tissue mimicking phantom and real tissues both in-vitro and in-vivo. The data is based on different numbers of lesions for each application. For the resected tissue and the EUS-elastography of pancreatic focal lesions, all recordings are done by the same observer, while the phantom inclusions are done by two observers. The fact that the data on surgical specimens and pancreatic lesions in this study are collected from individual lesions and patients while the phantom inclusions represent only four different "cases" also serves to limit the variability in the phantom SR measurements. The same strain elastography system was used for all scans (Real-Time elastography, RTE), but both scanners and software were upgraded between the first and the last recording.

\section{Recommendations for measuring SR in soft tissues}

Based on our experience with strain elastography in-vitro and in-vivo we propose the following advice to obtain relevant and reproducible elastograms:

- Select a US-plane where the lesion can be compared with relevant reference tissue, where both may be exposed to similar stress.

- Reference area should be applied to relevant tissue and be of similar size if possible. A too small reference area may cause sampling error.

- Select lesion and reference tissue at approximately same distance from the stress source if possible. In external imaging, the stress source is most often equivalent with the probe.

- Avoid sliding anatomical surfaces and visible vessels or ducts as part of the reference area.

- Measure SR in the same phase of compression or decompression when several measurements are being recorded.

- Measure at least three repeated SR - use the median

\section{Conclusions}

SR measurements are useful for quantifying local differences in tissue strain. We have shown that SR as a semi-quantitative method, has increased variability when targeted on a tissue mimicking phantom, resected biological tissue and vital tissue examined by endoscopic ultrasonography, respectively. Target area characteristics may limit the method's accuracy in clinical applications. SR also depends on similar stress application in reference and lesion areas. Homogeneous tissue facilitates SR measurements. Soft lesions have low SR values and relatively low variability, while harder lesions have higher SR measurement and variability. Attenuation of stress with depth and tissue inhomogeneity and anisotropy increase strain variability.

\footnotetext{
Acknowledgments: The studies that are reported data from in this paper are all conceived at Department of Medicine and the National Centre for Ultrasound in Gastroenterology (NCGU), Haukeland University Hospital. The funding of equipment and salaries were provided by the Medical Department, Haukeland University Hospital and University of Bergen, Institute of clinical Medicine. The studies were supported by MedViz an interdisciplinary research cluster from Haukeland University Hospital, University of Bergen and Christian Michelsen Research AS. We thank professor emeritus Svein Ødegaard for his tutoring and guidance and specialist nurse Eva Fosse for her great support during data acquisition. We will also express our gratitude to the support and guidance we have been given from the application specialists in Hitachi Medical Corporation, Europe. Hitachi Medical Corporation has not provided equipment for free nor has had any influence on the planning, execution or reporting in the studies reported.
} 
Author Contributions: Havre, R.F. and Nesje, L.B. and Gilja O.H. planned the studies and provided the equipment. Nesje L.B. and Gilja O.H. supervised in the collection of data, analyses and writing of the manuscript. Havre R.F. collected and analyzed the data and wrote the manuscript. Waage J.E.R. was a consultant in performing the elastography study on surgical specimens and pancreatic lesions with Real-Time Elastography. He also prepared the manuscript. Mulabecirovic A. presented preliminary data at DDW 2016 and prepared the manuscript.

Conflicts of Interest: The authors declare no conflict of interest. The founding sponsors had no role in the design of the study; in the collection, analyses, or interpretation of data; in the writing of the manuscript, and in the decision to publish the results.

\section{Appendix A}

S1: Excel file containing data based on the median SR for different phantom inclusions and etiologies (see it in the supplementary file)

\section{References}

1. Ophir, J.; Cespedes, I.; Ponnekanti, H.; Yazdi, Y.; Li, X., Elastography: a quantitative method for imaging the elasticity of biological tissues. Ultrason Imaging 1991, 13, (2), 111-34.

2. Aghajani, A.; Haghapanhi, M.; Nikazad, T., The ultrasound elastography inverse problem and the effective criteria. Proc Inst Mech Eng H 2013, 11, (227), 1203-12.

3. Ueno, E.; Umemoto, T.; Bando, H.; Tohno, E.; Waki, K.; Matsumura, T., New Quantitative method in Breast elastography: Fat lesion ratio (FLR). In Radiological Societyof North America Scientific Assembly and Annual Meeting, Chicago, Illinois, USA, 2007.

4. Diamond, L.; Murphy, M. Lesson: Stress, Strain and Hooke's Law. https://www.teachengineering.org/view lesson.php?url=collection/van/lessons/van cancer lesson2/ van cancer_lesson2.xml (April 11 2018),

5. Krouskop, T. A.; Wheeler, T. M.; Kallel, F.; Garra, B. S.; Hall, T., Elastic moduli of breast and prostate tissues under compression. Ultrason Imaging 1998, 20, (4), 260-74.

6. Umemoto, T.; Ueno, E.; Matsumura, T.; Yamakawa, M.; Bando, H.; Mitake, T.; Shiina, T., Ex vivo and in vivo assessment of the non-linearity of elasticity properties of breast tissues for quantitative strain elastography. Ultrasound Med Biol 2014, 40, (8), 1755-68.

7. Ponnekanti, H.; Ophir, J.; Huang, Y.; Cespedes, I., Fundamental mechanical limitations on the visualization of elasticity contrast in elastography. Ultrasound Med Biol 1995, 21, (4), 533-43.

8. Havre, R. F.; Waage, J. R.; Gilja, O. H.; Odegaard, S.; Nesje, L. B., Real-Time Elastography: Strain Ratio Measurements Are Influenced by the Position of the Reference Area. Ultraschall Med 2011, 33, (6), 10.

9. Le Sant, G.; Ates, F.; Brasseur, J. L.; Nordez, A., Elastography Study of Hamstring Behaviors during Passive Stretching. PLoS One 2015, 10, (9), e0139272.

10. Bamber, J.; Cosgrove, D.; Dietrich, C. F.; Fromageau, J.; Bojunga, J.; Calliada, F.; Cantisani, V.; Correas, J. M.; D'Onofrio, M.; Drakonaki, E. E.; Fink, M.; Friedrich-Rust, M.; Gilja, O. H.; Havre, R. F.; Jenssen, C.; Klauser, A. S.; Ohlinger, R.; Saftoiu, A.; Schaefer, F.; Sporea, I.; Piscaglia, F., EFSUMB guidelines and recommendations on the clinical use of ultrasound elastography. Part 1: Basic principles and technology. Ultraschall Med 2013, 34, (2), 169-84.

11. Havre, R. F.; Odegaard, S.; Gilja, O. H.; Nesje, L. B., Characterization of solid focal pancreatic lesions using endoscopic ultrasonography with real-time elastography. Scand J Gastroenterol 2014, 49, (6), 742-51. 
411 12. Iglesias-Garcia, J.; Larino-Noia, J.; Abdulkader, I.; Forteza, J.; Dominguez-Munoz, J. E., Quantitative endoscopic ultrasound elastography: an accurate method for the differentiation of solid pancreatic masses. Gastroenterology 2010, 139, (4), 1172-80.

13. Dawwas, M. F.; Taha, H.; Leeds, J. S.; Nayar, M. K.; Oppong, K. W., Diagnostic accuracy of quantitative EUS elastography for discriminating malignant from benign solid pancreatic masses: a prospective, single-center study. Gastrointest Endosc 2012, 76, (5), 953-61.

14. Riishede, I.; Ewertsen, C.; Carlsen, J.; Petersen, M. M.; Jensen, F.; Nielsen, M. B., Strain Elastography for Prediction of Malignancy in Soft Tissue Tumours--Preliminary Results. Ultraschall Med 2015, 36, (4), 369-74

16. Itoh, A.; Ueno, E.; Tohno, E.; Kamma, H.; Takahashi, H.; Shiina, T.; Yamakawa, M.; Matsumura, T.,

17. Giovannini, M.; Thomas, B.; Erwan, B.; Christian, P.; Fabrice, C.; Benjamin, E.; Genevieve, M.; Paolo, A.; Pierre, D.; Robert, Y.; Walter, S.; Hanz, S.; Carl, S.; Christoph, D.; Pierre, E.; Jean-Luc, V. L.; Jacques, D.; Peter, V.; Andrian, S., Endoscopic ultrasound elastography for evaluation of lymph nodes and pancreatic masses: a multicenter study. World J Gastroenterol 2009, 15, (13), 1587-93.

18. Waage, J. E.; Rafaelsen, S. R.; Borley, N. R.; Havre, R. F.; Gubberud, E. T.; Leh, S.; Kolbro, T.; Hagen, K. K.; Eide, G. E.; Pfeffer, F., Strain Elastography Evaluation of Rectal Tumors: Inter- and Intraobserver Reproducibility. Ultraschall Med 2015. diagnosis: strain ratio vs 5-point scale. Acad Radiol 2010, 17, (10), 1227-33.

21. Lu, Y.; Chen, L.; Li, C.; Chen, H.; Chen, J., Diagnostic utility of endoscopic Saftoiu, A.; Vilmann, P.; Gorunescu, F.; Janssen, J.; Hocke, M.; Larsen, M.; Iglesias-Garcia, J.; Arcidiacono, P.; Will, U.; Giovannini, M.; Dietrich, C. F.; Havre, R.; Gheorghe, C.; McKay, C.; Gheonea, D. I.; Ciurea, T.; European, E. U. S. E. M. S. G., Efficacy of an artificial neural network-based approach to endoscopic ultrasound elastography in diagnosis of focal pancreatic masses. Clin Gastroenterol Hepatol 2012, 10, (1), 84-90 e1. ultrasonography-elastography in the evaluation of solid pancreatic masses: a meta-analysis and systematic review. Med Ultrason 2017, 19, (2), 150-158. adenomas and adenocarcinomas. Colorectal Dis 2015, 17, (2), 124-31. 